\title{
Brain Tumor Extraction from MRI Images Using MATLAB
}

\author{
Pranay Rangne ${ }^{1}$, Prof. R.M.Bhombe ${ }^{2}$, Prof. A.S.Welankiwar ${ }^{3}$ \\ Department of Electrical Engineering \\ Guru Nanak Institute of Engineering and Technology, Nagpur, Maharashtra, India.
}

\begin{abstract}
Computerized or Automatic detection of tumors in medical images is motivated by the necessity of high accuracy when dealing with a human life. The computer assistance is also demanded in medical institutions because it could improve the results of disease identification and negative cases should be very low. So, the Processing of Magnetic Resonance Imaging (MRI) images is one of the techniques to diagnose the brain tumor. This paper describes the strategy to detect and extract brain tumor from patient's MRI scanned images. In this the Steps includes are pre-processing, segmentation, morphological operation, watershed segmentation and calculation of the tumor area and determination of the tumor location and this Application is Developed using Matrix Laboratory (MATLAB).
\end{abstract}

Keywords:- Brain tumor, Morphological- Operation, Water-shed segmentations, MATLAB.

\section{INTRODUCTION}

Brain Tumor, it is defined as an abnormal growth of cells in the brain tissues it is called brain tumor There are two main types of tumors such as:

$>$ Malignant(cancerous) tumor.

$>$ Benign (non-cancerous) tumor.

The Malignant Tumor is divided into two parts

Primary tumor

Secondary tumor

In Primary tumor, it starts within the brain, it is also called as Original or first tumor in the body. In Secondary tumor, it the most common tumor which spreads outside the brain. Primary tumor forms secondary tumor. There are also other types other than this but all tumors have same symptoms that vary depending on the part of brain affected. The symptoms can be headaches, seizures, having problems in visions, vomiting and mental changes are basic symptoms. Sometime The headache gets worse in the morning and goes away with vomiting. Some other symptoms include difficulty in walking and speaking or having sensations.

To diagnose the tumor, multiple Process is followed including MRI scan.

MRI scan which means Magnetic resonance imaging, which is a technique to produce high quality images of human body structure, it is similar like X-rays, but having more details and when we use MRI scan on brain it provides rich information of brain which helps to diagnosis and biomedical researching. The scanned values of MRI are more magnified by its automated and accurate classification.

There is also and Wavelet transform tool.

This tool is very affective and helpful for identifying the brain internal parts clearly, it has also a feature where it allows analysis of various levels of resolutions images by its multi- resolution analytic feature.

But there is a drawback, it requires large storage and computationally expensive. So, overcome with this problem PCA is used.

The principal component analysis (PCA) is effective to reduces the data which also reduces the computational cost to analysing fresh new data.

After this, there is one more problem of classifying input data arises. In past recent years, researchers introduce lots of goal to overcome with this problem. There are two categories.

\section{$>$ Supervised \\ $>$ Unsupervised}

In Supervised it includes support vector machine (SVM) and Knearest neighbors (K- NN).

In Unsupervised it includes self- organization feature map (SOFM) and fuzzy c-means.

These two categories are helpful and gives good result, but supervised method performs better than unsupervised method have more accuracy compared to other one. But both having 95\% accuracy, and if we use other similar methods to achieve best result but all are end up with $95 \%$ accuracy.

\section{LITERATURE SURVEY}

While working on the Project I had encountered with many problems and to overcome this problem I have done lots of surveys reads multiple books and talked with doctors and search on internet to solve the problems facing while completing the Project, I have found some books which helps me overcome the problems some of them are Bhagwat et al, introduced a paper where he shows the comparison of Fuzzy C-means, K-means and Hierarchical 
clustering formula/algorithms which detects the brain tumor, These Three clustering algorithms are tested with MRI brain image with format (.jpg, .png, .bmp etc) and with DICOM image. After testing it founds that DICOM images produce more efficient result as compared with computer images format (.jpg,.png,.bmp etc). Whereas time taken in hierarchical clustering less than fuzzy c-means to detect brain tumor. And in other hand K- means produces more accurate result as compared to fuzzy c- means and hierarchical clustering. The Aim is to detect tumor in brain so to achieve the aim, it involves multiple stages like preprocessing images, extractions properties segmentation and classification.

The second one is Rajesh patil etal paper which help me to achieve more in Depth knowledge for extracting tumor, he introduced a method which detects and extract the tumor via computerized software called MATLAB, the software requires and Image for a person's brain who is having brain tumor and by using the software it will extract the tumor digitally. It uses MRI Images which is more convenient and having depth image.

The method involves noise removing, segmentation and morphological operations based on image processing.

\section{SYSTEM ANALYSIS}

\section{Practice Development}

As proposed in state of the art one of the items develop was that of preprocessing to extract information from the magnetic resonance image and indeed that was the basis of where the project is developed. However, it had also proposed to make filtered image to study but as seen later this is not efficient for the type of image being treated. So, based on the above the project development around the extraction of features by pre-processing and application of a transform that although it will not directly affect the image allows to acquire relevant data for the further study of the image. Morphological operators also were used for extraction of the desired region to study (tumor), then it proposed an algorithm for the extraction of the tumor. Each function is observed properly to detect tumor and calculate the tumor.

This is Described in the Response of MATLAB.

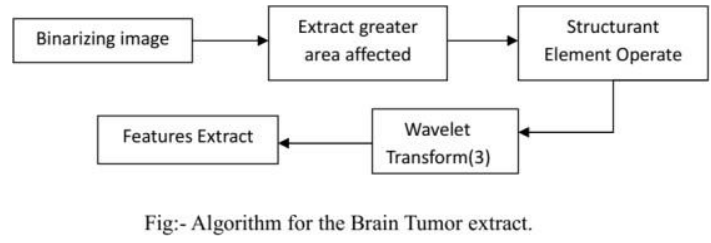

Fig 1

\section{$>$ Binarizing image}

binarizing the image to what it does is it make to a single palette or either black or white is, that in order to abruptly remove all information of the image that is not used for analysis. To accomplish this in Matlab the following command line is used:
$\mathrm{I}=$ handles.img; img $=\mathrm{im} 2 \mathrm{bw}(\mathrm{I}, 0.6)$;

img1 = bwareaopen(img,80);

Where ' $I$ ' is the original image and the im $2 \mathrm{bw}$ command acts as a function to convert any image to black and white, the input parameters of the previous command is the object (image) to be binarizing and the level of binarization of that transformation. After that also you choose to use an operator morphologic opening. The result in this section is an image like shows in the figure $2 \mathrm{~b}$.

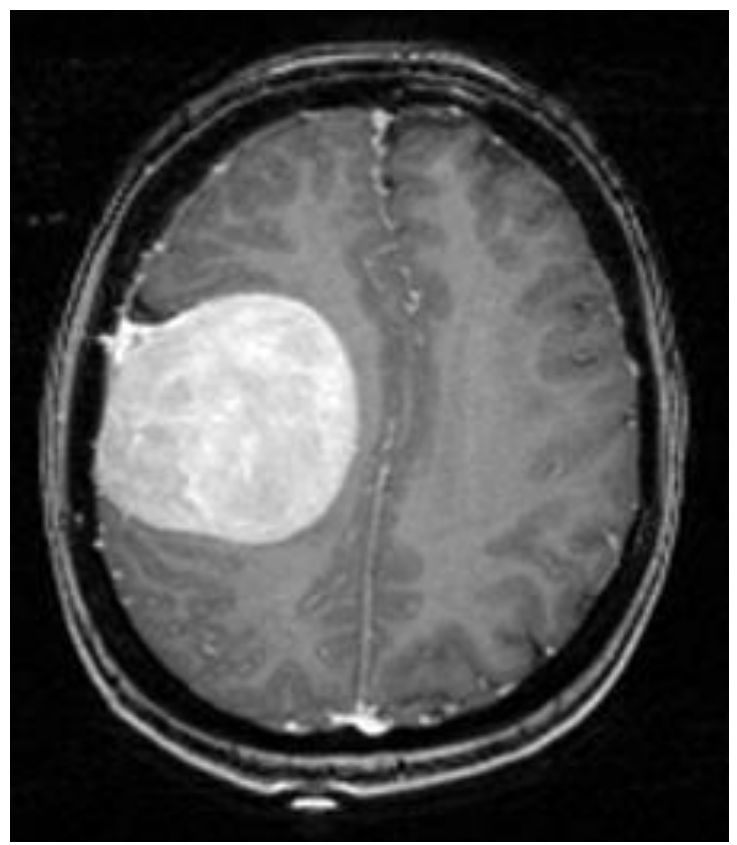

(a)

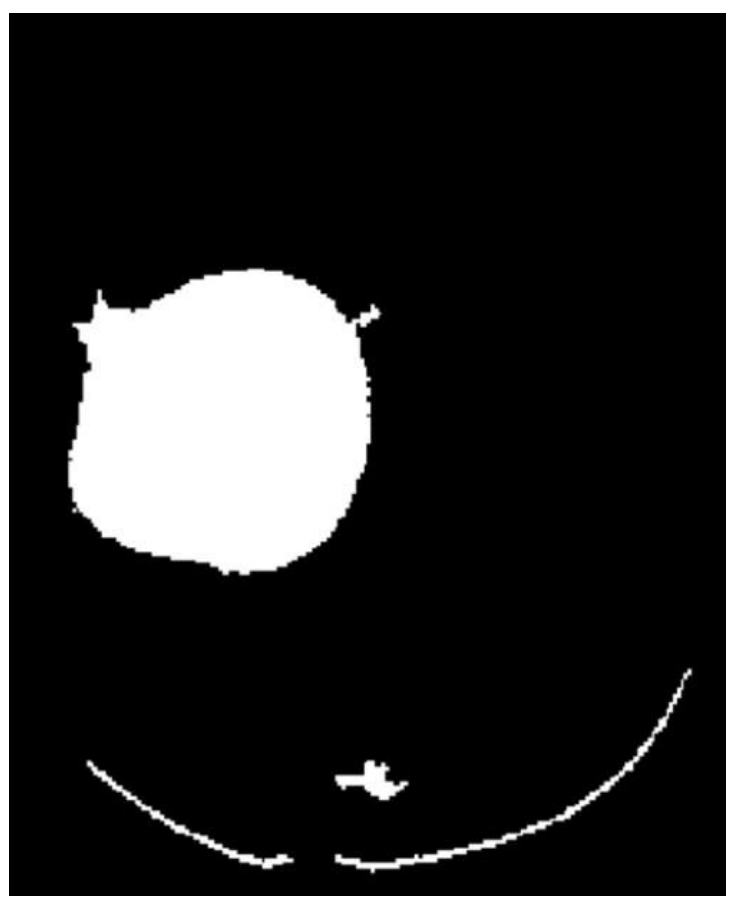

(b)

Fig 2:- a) Oringinal Patient MRI b) bonarized image with opening operator 
However, this type of binarization with the opening let some unwanted elements, then it was decided to use another method if allowed to remove only the tumor area. In this case the following command line is used:

The result is shows in the fig 3 .

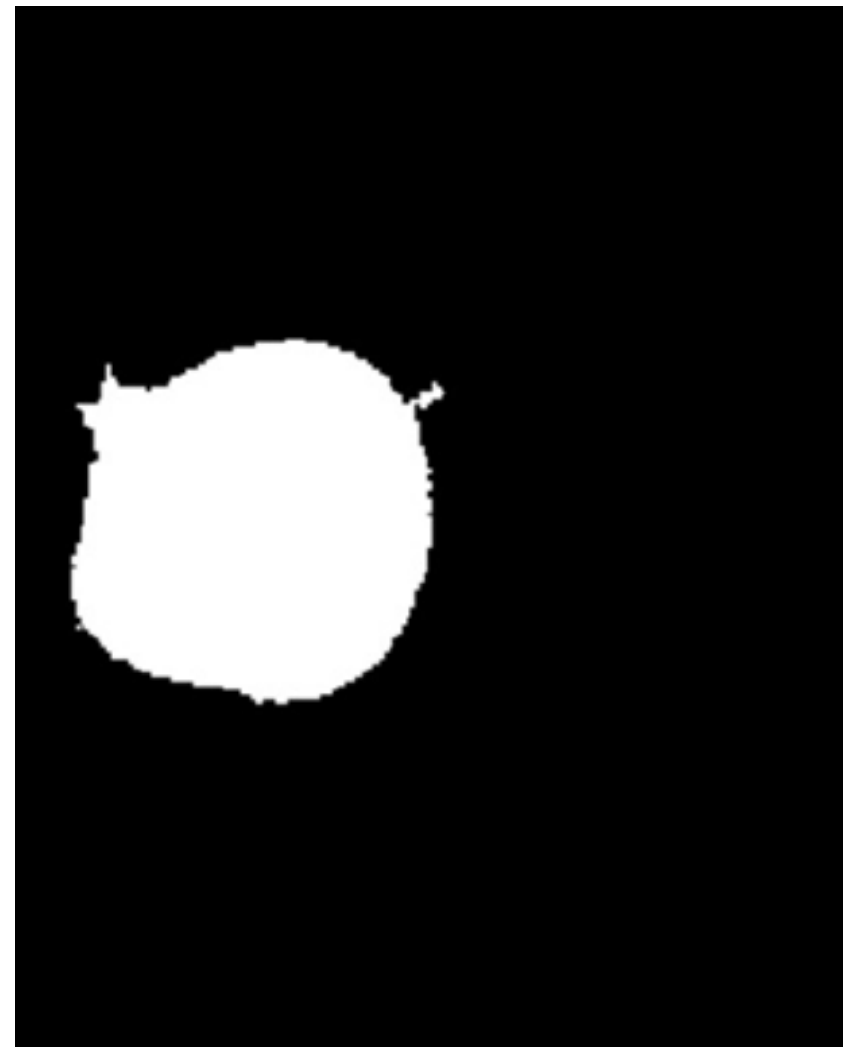

Fig 3:- Reion affected

Extracting the greater areaaffected

Var name =bwname (img); Var region = regionprops(name, 'Solidity', 'Area'); Var density = [region. Solidity]

Var volume $=$ [region. Area]

Var density_area $=$ density $>0.3 ;$ Var max_area $=$ max(volume(density_area));

Var tumor_name $=$ find $($ volume $==$ max_area $)$;

Var tumorD = ismember(name,tumor_name);

with "regionprops" command measures those image components. This command requires two entries: the components "label" and its characteristics, in this case 'solidity' are chosen and 'area'. The reason for this is because the image fastness tumor will be much greater than the strength of the rest of the image then this will help remove those parts of the image. Then the density of this region is calculated by the "stats" command. The same is done for the area.

Then you can that have regions in the image with perhaps the same density and area of the tumor but that are no parts of tumor, then it must establish a margin of radio which is started to detect that whether or not a tumor. The following command lines do a type of operations to calculate the maximum area with densities but limited by a value of 0.3 , thus values below this number are not operated

\section{Structuring element operate}

This functional block in the region of Tumor simply the anteiror section is operated and operates with a morphological (square) operator.

var tumorD = ismember(name, tumor_name); var $s q=\operatorname{strel}($ 'square', 1);

Wavelet transform (WT) it a technique of window adjusting with variable size.So, it helps to store values of both time and frequency signals. The Signal Analysis is shown in fig 4. It is an Process fo development of signals.

There are different advantages also in WT such as It adopts 'Scale' instead of 'frequency' which was used in old and traditional applications.

The time scale is more powerful and natural as compared to frequency it has also a different way to view data compared to frequency time.

And large/small scale is also easily understands compared to high/low frequency so it is important and it is used.

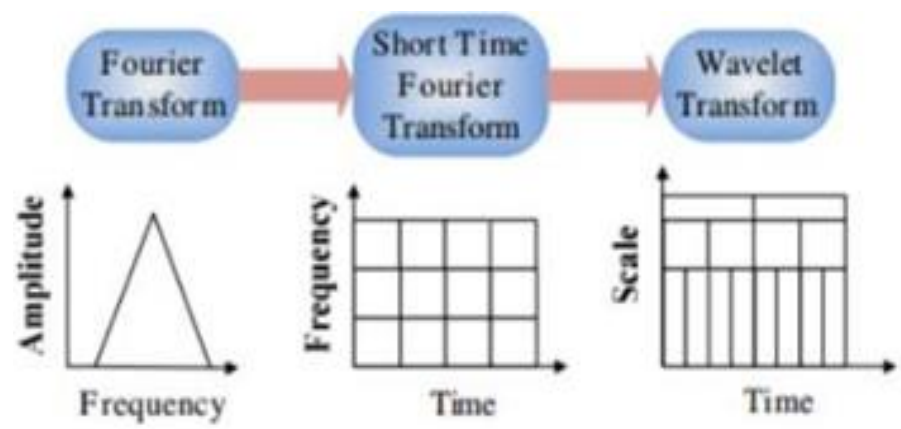

Fig 4:- The development of signal analysis 


\section{RESULT}

Bain tumor Extraction from MRI Images using MATLAB is shown in Fig 5.

The Screenshot shows all the type of Images process images which helps to Detected tumor. Thus, we get the expected result of tumor.

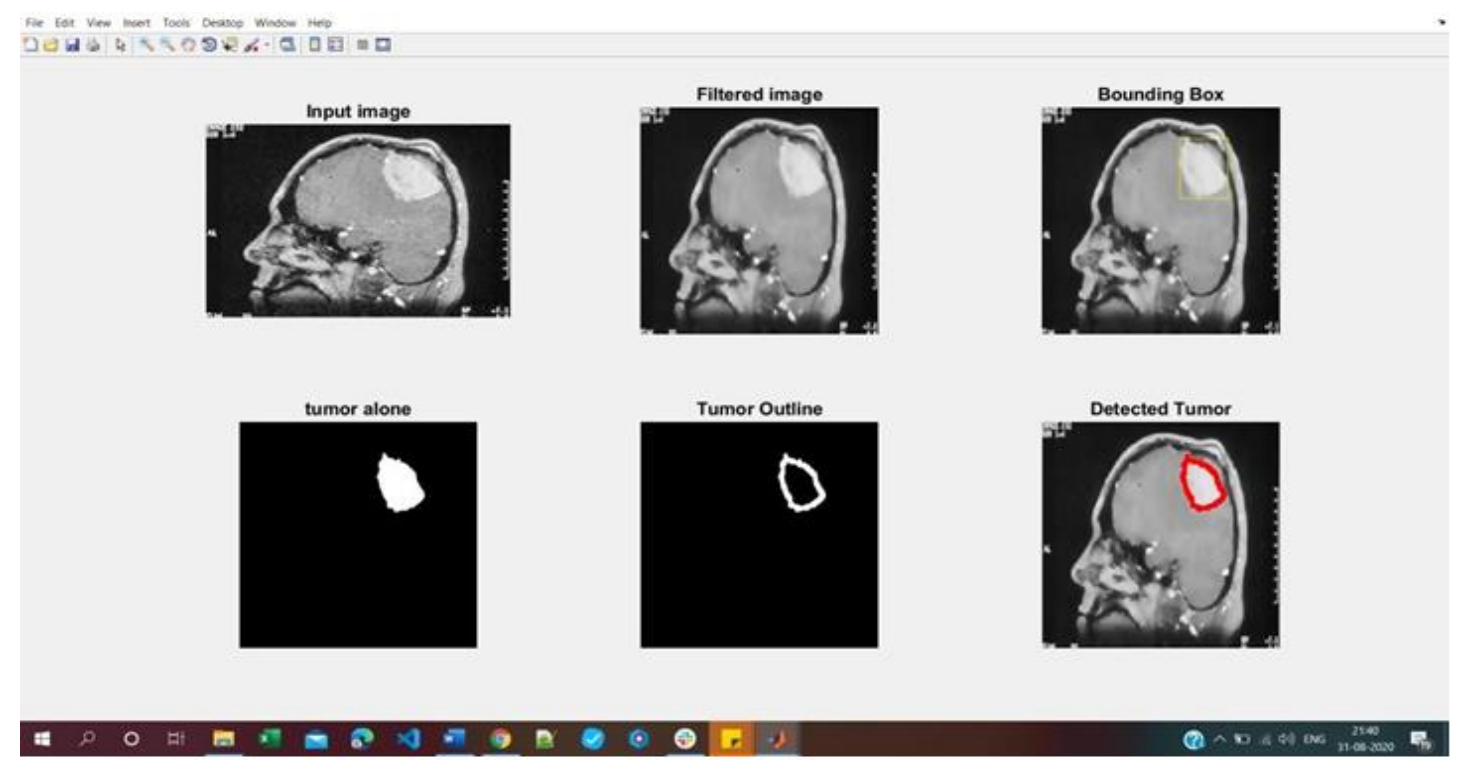

Fig 5:- Multi-stages of Brain Tumor extraction from MRI in MATLAB

\section{CONCLUSION}

Spatial filtering and frequency filtering are not recommended for treatment in medical imaging such as CAT scans because they steal vital information for diagnosis to the image because the filtered that are made are made on the whole picture, however with morphological operations can work the image pixel by pixel or can perform a segmentation of the image to separate objects and can identify them without any data loss problem after completing treatment. Sometimes applications of digital image processing are simply summarized do transformations on the image but no analysis can become wider leveraging the benefits of tools like MATLAB as was evident offers many features that can become relevant to the analysis of the images

\section{REFERENCES}

[1]. Zhang, Y., L. Wu, and S. Wang, "Magnetic resonance brain image classification by an improved artificial bee colony algorithm," Progress in Electromagnetics Research, Vol. 116, 65-79, 2011.

[2]. W. Gonzalez, "Digital Image Processing", 2nd ed. Prentice Hall, Year of Publication 2008, Page no 378.

[3]. S. Murugavalli, V. Rajamani, "A high speed parallel fuzzy c-mean algorithm for brain tumour segmentation"," BIME Journal", Vol. no: 06, Issue (1), Dec., 2006

[4]. Mohsin, S. A., N. M. Sheikh, and U. Saeed, "MRI induced heating of deep brain stimulation leads: Effect of the air- tissue interface, Progress in Electromagnetics Research, Vol. 83, 81- 91, 2008.
[5]. Golestanirad, L., A. P. Izquierdo, S. J. Graham, J. R. Mosig, and C. Pollo, "Effect of realistic modeling of deep brain stimulation on the prediction of volume of activated tissue," Progress in Electromagnetics Research, Vol. 126, 1- 16, 2012.

[6]. Mohsin, S. A., "Concentration of the specific absorption rate around deep brain stimulation electrodes during MRI," Progress in Electromagnetics Research, Vol. 121, 469-484, 2011.

[7]. Oikonomou, A., I. S. Karanasiou, and N. K. Uzunoglu, "Phasedarray near field radiometry for brain intracranial applications," Progress in Electromagnetics Research, Vol. 109, 345-360, 2010.

[8]. Scapaticci, R., L. Di Donato, I. Catapano, and L. Crocco, "A feasibility study on microwave imaging for brain stroke monitoring," Progress

in Electromagnetics Research B, Vol. 40, 305-324, 2012.

[9]. Asimakis, N. P., I. S. Karanasiou, P. K. Gkonis, and N. K. Uzunoglu, "Theoretical analysis of a passive acoustic brain monitoring system," Progress in Electromagnetics Research B, Vol. 23, 165-180, 2010.

[10]. Chaturvedi, C. M., V. P. Singh, P. Singh, P. Basu, M. Singaravel, R. K. Shukla, A. Dhawan, A. K. Pati, R. K. Gangwar, and S. P. Singh. "2.45 GHz (CW) microwave irradiation alters circadian organization, spatial memory, DNA 